\title{
Characteristics and associated risk factors of diverticular disease assessed by magnetic resonance imaging in subjects from a Western general population
}

\author{
Corinna Storz ${ }^{1}$ - Theresa Rothenbacher ${ }^{1}$. Susanne Rospleszcz ${ }^{2}$. Jakob Linseisen ${ }^{2,3} \cdot$ Helmut Messmann ${ }^{4}$. \\ Carlo N. De Cecco ${ }^{5}$. Jürgen Machann ${ }^{6,7,8}$ • Roberto Lorbeer ${ }^{9}$. Lena S. Kiefer ${ }^{1}$ - Elke Wintermeyer ${ }^{10}$ - Sophia D. Rado ${ }^{1}$. \\ Konstantin Nikolaou ${ }^{1}$ - Stefanie Elser ${ }^{1}$ - Wolfgang Rathmann ${ }^{11}$ • Maximilian F. Reiser ${ }^{9}$. Annette Peters ${ }^{2,12,13,14}$. \\ Christopher L. Schlett ${ }^{15} \cdot$ Fabian Bamberg $^{1}$
}

\begin{abstract}
Objectives Diverticular disease represents an increasing pathology and healthcare burden worldwide. Our aim was to study the prevalence, extent and distribution of asymptomatic diverticular disease assessed by magnetic resonance imaging (MRI) in a sample of a Western population.

Methods Subjects from a population-based cohort study who underwent 3-T MRI were analyzed for the prevalence and extent of diverticula of the colon using an isotropic VIBE-Dixon gradient-echo sequence. The extent of diverticular disease was categorized according to the number of diverticula in each colonic segment. Univariate and adjusted analyses were performed to assess associated characteristics and risk factors.

Results Among 393 subjects included in the analysis (56.4 \pm 9.2 years, 57.5\% males), 164 (42\%) had diverticular disease, with the highest prevalence in the left-sided colonic segments (93\% diverticular disease in the descending and sigmoid segment). Subjects with advanced diverticular disease were older (62.1 vs. 54.4 years) and had a higher body mass index (BMI), LDL cholesterol levels and systolic blood pressure $\left(30.2 \pm 5.1\right.$ vs. $27.8 \pm 4.9 \mathrm{~kg} / \mathrm{m}^{2}, 149.8 \pm 29.3 \mathrm{vs} .135 .2 \pm 32.9 \mathrm{mg} / \mathrm{dl} \mathrm{and} 128.2 \pm$ 14.1 vs. $118.4 \pm 16.1 \mathrm{mmHg}$, respectively; all $p>0.003$ ) compared with subjects without diverticular disease. In contrast, no significant correlation could be found for gender, physical activity, smoking status and alcohol consumption (all $p>0.31$ ). Intra-
\end{abstract}

Fabian Bamberg

fabian.bamberg@uni-tuebingen.de

1 Department of Diagnostic and Interventional Radiology, University Hospital Tuebingen, Hoppe-Seyler Str. 3,

72076 Tuebingen, Germany

2 Institute of Epidemiology, Helmholtz Centre Munich, German Research Center for Environmental Health, Neuherberg, Germany

3 University Center of Health Sciences at Klinikum Augsburg (UNIKA-T), Ludwig Maximilian University of Munich, Augsburg, Germany

4 Department of Internal Medicine III, Klinikum Augsburg, Augsburg, Germany

5 Department of Radiology, Medical University of South Carolina, Charleston, SC, USA

6 Section on Experimental Radiology, Department of Diagnostic and Interventional Radiology, University Hospital Tuebingen, Tuebingen, Germany
7 Institute for Diabetes Research and Metabolic Diseases (IDM) of the Helmholtz Center Munich at the University of Tuebingen, Tuebingen, Germany

8 German Center for Diabetes Research (DZD), Tuebingen, Germany

9 Department of Radiology, Ludwig Maximilian University Hospital, Munich, Germany

10 Siegfried Weller Institute for Trauma Research, BG Trauma Center Tübingen, Eberhard Karls University Tübingen, Tübingen, Germany

11 Department of Biometry and Epidemiology, German Diabetes Center, Düsseldorf, Germany

12 German Center for Cardiovascular Disease Research (DZHK e.V.), Munich, Germany

13 Institute for Cardiovascular Prevention, Ludwig Maximilian University-Hospital, Munich, Germany

14 Chair of Epidemiology, Ludwig Maximilian University, Munich, Germany

15 Department of Radiology, Diagnostic and Interventional Radiology, University of Heidelberg, Heidelberg, Germany 
rater reliability was excellent for all colonic segments (intra-class correlation $[\mathrm{ICC}]=0.99-1.00$ ), and inter-rater reliability was excellent for left- and right-sided colonic segments $(\mathrm{ICC}=0.84-0.97)$.

Conclusions These findings provide insights into the disease mechanism of asymptomatic diverticular disease and may help to improve prevention of diverticulosis and its associated complications.

Key Points

- Overall prevalence of asymptomatic diverticular disease assessed by MRI was 42\%, affecting predominantly the left-sided colon.

- Asymptomatic diverticular disease was associated with age and cardiometabolic risk factors.

- Magnetic resonance imaging reveals insights into the pathophysiologic mechanism of asymptomatic diverticular disease.

Keywords Diverticular disease $\cdot$ Magnetic resonance imaging $\cdot$ Colon $\cdot$ Epidemiology

\section{Abbreviations}

BMI Body mass index

CT Computed tomography

CTC Computed tomography colonography

ICC Intraclass correlation coefficient

LDL Low-density-lipoprotein

MRI Magnetic resonance imaging

OGTT Oral glucose tolerance test

OR Odds ratio

\section{Introduction}

Colonic diverticular disease refers to multiple outpouches from the colonic lumen due to weakness of the muscular wall in the mucosa and harbors the risk of acute and/or chronic inflammatory changes potentially leading to lifethreatening complications such as bleeding, bowel perforation and peritonitis [1-4]. Several studies previously reported that the prevalence of diverticular disease increases with age ranging from $32.6 \%$ in 50-59-year-old individuals to $71.4 \%$ by the age of 80 years and older. Since the elderly population is growing, diverticular disease represents an increasing pathology and healthcare burden worldwide, especially in Western and industrialized countries [5].

The exact prevalence of asymptomatic diverticular disease is difficult to assess. Initial results of several largescale clinical studies using colonoscopy or computed tomography (CT) indicate that the prevalence of diverticulosis is still increasing with a substantial set of risk factors promoting the development of diverticular disease [5-7]. Diverticular disease seems to be associated with various underlying factors such as colonic wall alterations or disordered motility, dietary habits, obesity, smoking and various medications [2, 3, 8, 9]. Furthermore, early research indicates a genetic predisposition to the development of diverticular disease $[10,11]$. In approximately $10-25 \%$ of individuals with diverticulosis, inflammatory alterations with diverticulitis will occur $[4,12]$. This is partially in contrast to earlier clinical data in asymptomatic patients who underwent colonoscopies, indicating that only $4 \%$ of the individuals with asymptomatic diverticulosis developed later diverticulitis, while in younger patients, a higher risk of progression of diverticular disease was observed, highlighting the missing information about the pathophysiologic mechanism of asymptomatic and symptomatic diverticular disease [13]. However, studies about asymptomatic diverticular disease investigating the extent and distribution are scarce, particularly in a general population.

Magnetic resonance imaging (MRI) represents a noninvasive imaging modality without the need of radiation exposure, also established for the evaluation of the gastrointestinal tract, including diverticular disease [14]. Regarding the diagnostic modalities of inflammatory diseases of the gastrointestinal tract, previous research showed that MRI is a feasible alternative modality to $\mathrm{CT}$ in the diagnosis and evaluation of acute colonic diverticulitis with high sensitivity and specificity [14-18]. However, there are no studies analyzing the presence and extent of asymptomatic diverticulosis by MRI.

Given the clinical relevance and still limited understanding of the associated pathophysiology of diverticulosis and diverticular disease, studies in larger populations are necessary to assess risk factors for the development of diverticular disease and potentially develop tailored prevention strategies. Therefore, the purpose of this study was to evaluate the characteristics of asymptomatic diverticular disease as assessed by MRI in an asymptomatic, population-based sample. Our hypothesis was that there are differences in the extent, colonic segmental distribution and distinct risk factors associated with asymptomatic diverticular disease.

\section{Material and methods}

\section{Study design and recruitment of participants}

This study was designed as a case-control study, embedded in a prospective cohort from the KORA study, as 
described elsewhere [19]. Participants were recruited from the follow-up of the KORA study and were enrolled in an MRI sub-study between June 2013 and September 2014 at the KORA study center [19]. In brief, subjects without prior cardiovascular disease invited for a re-assessment of the prospective cohort study and willingness to undergo MRI examination were eligible to also participate in the MRI study if no contraindications to MRI and administration of gadolinium were present. The following exclusion criteria were applied: $>72$ years of age, participants with prior cardiovascular diseases such as stroke, myocardial infarction or revascularization, cardiac pacemaker or implantable defibrillator, cerebral aneurysm clip, neural stimulator, any type of ear implant, ocular foreign body, any implanted device, pregnant or breastfeeding subjects or participants with claustrophobia, known allergy against gadolinium compounds or impaired renal function with a serum creatinine level $\geq 1.3 \mathrm{mg} / \mathrm{dl}$ [19].

The study was approved by the institutional review board, and all participants provided written informed consent. More details of the study design, inclusion and exclusion criteria as well as the MRI protocol have been provided elsewhere [19].

\section{Covariates}

In this study, baseline information on sociodemographic variables and risk factors were collected by comprehensive interviews and a medical examination in a standardized fashion and were prospectively defined [20]. Body mass index (BMI) was defined as the value from the mass of the participant in kilograms divided by their height squared in meters. Smoking was defined as never, exand current smoker. Alcohol consumption was defined according to the anamnesis as no $(0 \mathrm{~g} /$ day $)$, moderate (0.1-39.9 $\mathrm{g}$ /day for males and 0.1-19.9 $\mathrm{g}$ /day for females) and heavy alcohol consumption $(\geq 40 \mathrm{~g} /$ day for males and $\geq 20 \mathrm{~g}$ /day for females) [21]. Subjects were categorized as physically active (regular physical activity $\geq 1 \mathrm{~h} /$ week) or physically inactive (irregular physical activity $<1 \mathrm{~h} /$ week, almost no physical activity and no physical activity at all). An oral glucose tolerance test (OGTT) was administered to all participants, and established definitions of diabetes and prediabetes were applied [22]. Specifically, prediabetes was defined as impaired glucose tolerance and/or impaired fasting glucose; healthy controls were defined as having normal glucose metabolism by OGTT [23]. Blood pressure was measured in the course of the KORA health study examinations, independent of the time of MRI acquisition. Hypertension was defined as increased systolic blood pressure $\geq 140 \mathrm{mmHg}$, increased diastolic blood pressure $\geq 90 \mathrm{mmHg}$ or use of antihypertensive medication. Medication being antihypertensive by the most recent guidelines was defined as 'antihypertensive medication,' and lipid-lowering medication was defined as the routine intake of statins, fibrates or other lipid-lowering agents. Other established risk factors were collected in standardized fashion as part of the study design, as previously described [20].

\section{Magnetic resonance imaging protocol}

All participants underwent an identical imaging protocol on a 3-T Magnetom Skyra MRI (Siemens Healthineers), as detailed previously [20]. For the assessment of diverticular disease, a two-point T1-weighted isotropic VIBEDixon gradient-echo sequence of the abdomen was employed, with the following parameters: TR $4.06 \mathrm{~ms}$, TE 1.26, $2.49 \mathrm{~ms}$, flip angle $9^{\circ}$, partition thickness 1.7 $\mathrm{mm}$ and isotropic in-plane resolution $1.7 \mathrm{~mm}$. This sequence acquires a total of four contrasts based on the chemical shift effect of water- and fat-bound protons: (1) the in-phase, (2) the opposed phase, (3) the water-only phase and (4) the fat-only phase (Fig. 1).

\section{Magnetic resonance imaging analysis}

The MRI images were assessed by two independent readers (CS and TR, radiology trainees with 3 and 2 years of experience, respectively, supervized by FB) who were blinded to all clinical and demographic information about the participants. A colonic diverticulum was defined as an outpouch from the colonic lumen [4]. The colonic segments were defined by anatomical landmarks and divided into the cecum, ascending colon, transverse colon, descending colon and sigmoid colon [24]. Each reader recorded the number and location of the diverticula for each participant in the colonic segments using commercially available medical imaging viewer software (OsiriX, Pixmeo). In line with previous research applying CT colonography (CTC), a graded-scale system was used to classify the extent of colonic diverticula [25]: grade 1 $=$ no diverticular disease; grade $2=$ mild diverticular disease with at least 1 but $<6$ diverticula in at least one colonic segment; grade 3 = advanced diverticular disease with $\geq 6$ diverticula in at least one segment of the colon. Concerning the reliability, inter- and intra-rater variability was assessed by intraclass correlation coefficient (ICC) analysis in a subset of 60 and 40 participants, respectively. For the assessment of intra-rater variability, measurements on 40 randomly selected data sets were performed after a period of at least 6 months to reduce recall bias. Concerning the image quality, the MRI images were classified as high (no artifacts), moderate (at least one colonic segment with limited image quality due to, i.e., motion artifacts but still diagnostic for the 
Fig. 1 Example of MRI acquisition in a 57-year-old male with advanced diverticular disease in the sigmoid colon by coronary multiplanar reconstruction of the T1-weighted VIBE-Dixon isotropic gradientecho sequence in the in-phase (a), opposed-phase (b), fat-only (c) and water-only (d) images. The white arrows indicate the diverticula

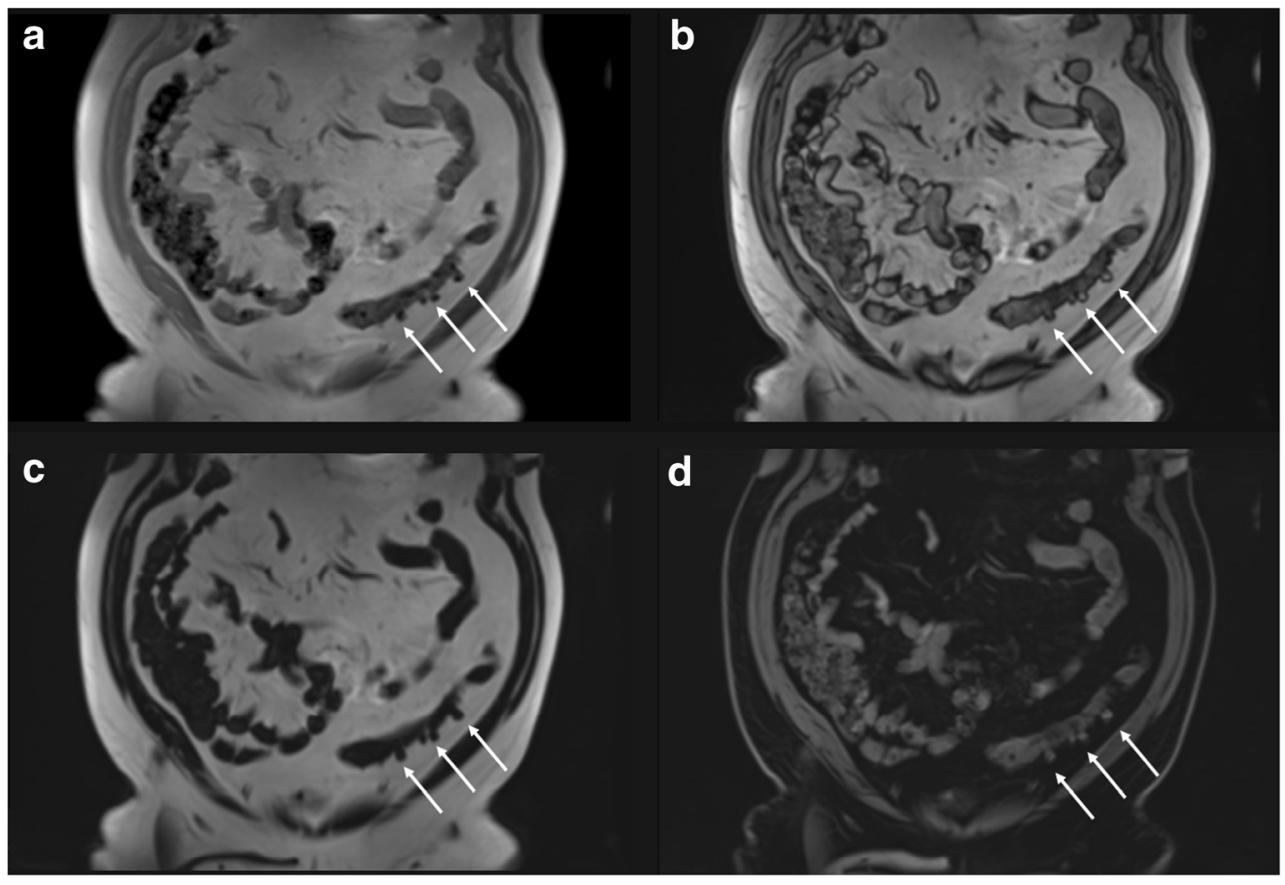

presence/absence of diverticular disease) or low (at least one colonic segment with impaired image quality precluding the diagnostic assessment of the presence of diverticular disease). Representative examples of participants with categorized diverticular disease are provided in Fig. 2; examples for the applied diverticular disease grading scale are given in Fig. 3 .

\section{Statistical analysis}

Subject demographics are reported as arithmetic means with standard deviation for continuous risk factors and as counts and percentages for categorical risk factors. Differences in risk factors between the stages of diverticular disease were assessed by one-way ANOVA and $\chi^{2}$-test, accordingly. Stages of diverticular disease according to the colonic segment are presented as counts and percentages. To analyze the association of risk factors for advanced diverticular disease, a logistic regression model containing the variables age, sex, BMI, systolic blood pressure, total cholesterol, LDL cholesterol and use of antihypertensive medication was calculated. Continuous variables were standardized to ensure the comparability of the resulting odds ratios (ORs). Two-sided $p$ values $<0.05$ are considered to indicate statistical significance. All computations were done with $\mathrm{R}$ version 3.4.1.

\section{Results}

Among 400 Caucasian participants enrolled in the MRI sub-study, 7 participants were excluded from the analysis because of missing data sets $(1.8 \%)$. Thus, 393 participants formed the study sample, being predominantly middle-aged males (mean age: $56.4 \pm 9.2$ years, $57.5 \%$ males). Demographics of the study population are provided in Table 1.

\section{Intra- and inter-rater reliability and image quality}

Intra-rater reliability was excellent for all colonic segments (ICC between 0.99 and 1.00), whereas inter-rater reliability was excellent for the right- and left-sided colonic segments (ICC between 0.84 and 0.97 ).

In 336 participants $(84.0 \%)$, the image quality was classified as high, in 57 participants $(14.3 \%)$ as moderate, and in 7 participants' data sets were missing $(1.8 \%)$, which were excluded from the analysis cohort.

\section{Diverticular distribution and severity}

Presence and distribution of diverticular disease are provided in Table 2. Absence of diverticular disease was observed in $229(58.3 \%)$ participants, while in 164 participant diverticular disease was present (prevalence, $41.7 \%$ ). Among subjects with diverticular disease, $24.2 \%$ had mild and $17.6 \%$ advanced diverticular disease. Within the colon, the prevalence of diverticular disease was highest in the descending and sigmoid colon $(41.0 \%$ and $42.8 \%$ for the descending and sigmoid colon, respectively), whereas the cecum had the lowest prevalence of diverticula $(0.7 \%)$. 
Fig. 2 Example of a 57-year-old female (a) and 64-year-old male (b) with advanced diverticular disease (white arrows) in the sigmoid colon in coronary and axial multiplanar reconstruction. $\mathrm{L}=$ liver, $\mathrm{K}=$ kidney, $\mathrm{S}=$ spleen
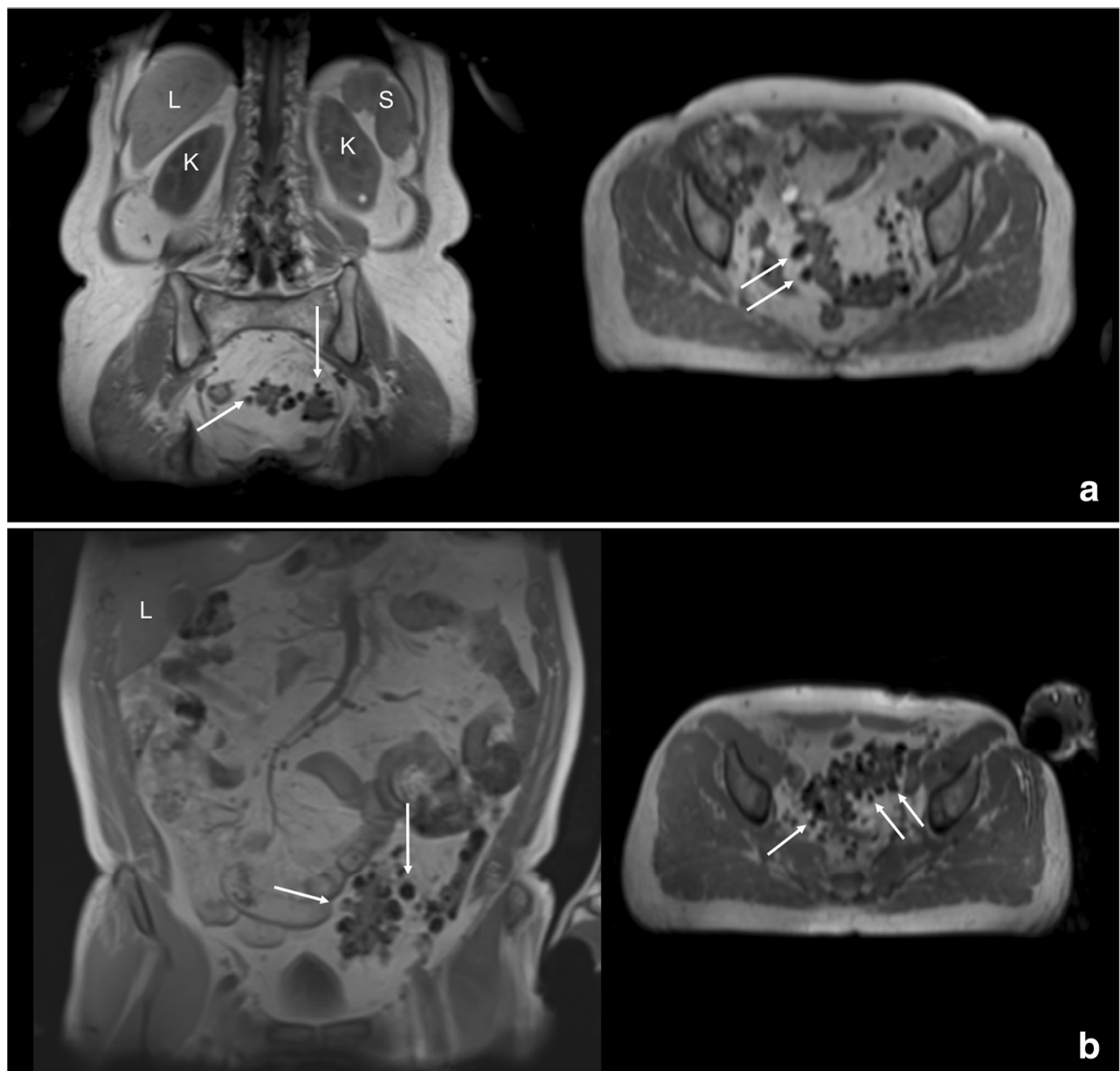

\section{Association of diverticular disease with risk factors}

Subjects with diverticular disease were significantly older, ranging from a mean age of $54.4 \pm 8.8$ years in participants without diverticular disease to a mean age of $62.1 \pm 7.7$ years in participants with advanced diverticular disease $(p \leq 0.001$,
Table 1). Furthermore, the grade of diverticular disease was significantly correlated with age (Fig. 4). Similarly, subjects with advanced disease had significantly higher BMIs than the control group (BMI: $27.8 \pm 4.9 ; 27.5 \pm 4.6$ and $30.2 \pm 5.1$ for participants without, with mild and advanced diverticular disease, respectively; $p=0.001$; Fig. 5).

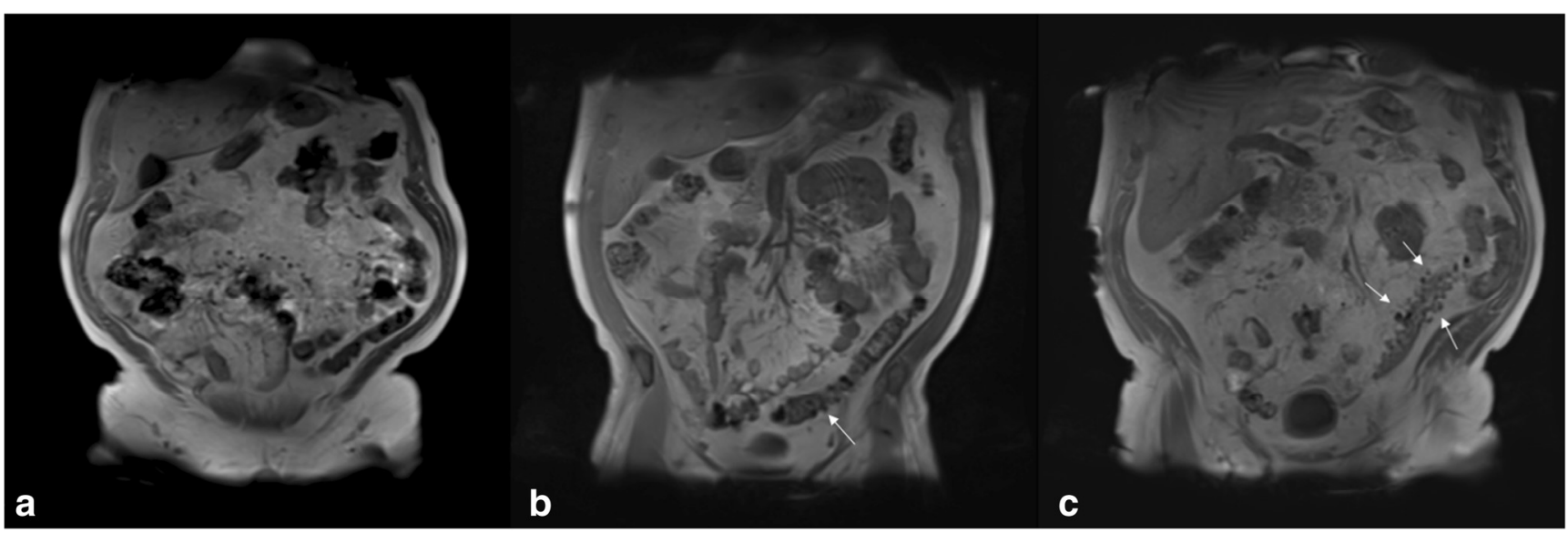

Fig. 3 Extent of diverticular disease according to the grading scale. a grade $1=$ no diverticular disease; $\mathbf{b}$ grade $2=$ mild diverticular disease with $<6$ diverticula (white arrows) in at least one colonic segment; c grade $3=$ advanced diverticular disease with $\geq 6$ diverticula in at least one segment of the colon 
Table 1 Patient demographic and risk factors according to the grade of diverticular disease

\begin{tabular}{|c|c|c|c|c|c|c|}
\hline Variable & All $n=393$ & $\begin{array}{l}\text { No diverticular } \\
\text { disease } n=229 \\
(58.3 \%)\end{array}$ & $\begin{array}{l}\text { Mild diverticular } \\
\text { disease } n=95 \\
(24.2 \%)\end{array}$ & $\begin{array}{l}\text { Advanced diverticular } \\
\text { disease } n=69 \\
(17.6 \%)\end{array}$ & $p$ value & $p$ value* \\
\hline Age (years) & $56.4 \pm 9.2$ & $54.4 \pm 8.8$ & $56.9 \pm 9.3$ & $62.1 \pm 7.7$ & $<0.001$ & $<0.001$ \\
\hline Males & $226(57.5 \%)$ & $128(55.9 \%)$ & $54(56.8 \%)$ & $44(63.8 \%)$ & 0.505 & 0.614 \\
\hline BMI $\left(\mathrm{kg} / \mathrm{m}^{2}\right)$ & $28.1 \pm 4.9$ & $27.8 \pm 4.9$ & $27.5 \pm 4.6$ & $30.2 \pm 5.1$ & 0.001 & 0.001 \\
\hline Normal & $105(26.7 \%)$ & $63(27.5 \%)$ & $31(32.6 \%)$ & $11(15.9 \%)$ & 0.008 & 0.015 \\
\hline Overweight & $168(42.7 \%)$ & $101(44.1 \%)$ & $42(44.2 \%)$ & $25(36.2 \%)$ & & \\
\hline Obese & $120(30.5 \%)$ & $65(28.4 \%)$ & $22(23.2 \%)$ & $33(47.8 \%)$ & & \\
\hline \multicolumn{7}{|l|}{ Smoking } \\
\hline Never-smoker & $144(36.6 \%)$ & $87(38.0 \%)$ & $35(36.8 \%)$ & $22(31.9 \%)$ & 0.394 & 0.435 \\
\hline Ex-smoker & $171(43.5 \%)$ & $96(41.9 \%)$ & $38(40.0 \%)$ & $37(53.6 \%)$ & & \\
\hline Smoker & $78(19.8 \%)$ & $46(20.1 \%)$ & $22(23.2 \%)$ & $10(14.5 \%)$ & & \\
\hline \multicolumn{7}{|l|}{ Diabetes status } \\
\hline Normoglycemic & $238(60.6 \%)$ & $140(61.1 \%)$ & $61(64.2 \%)$ & $37(53.6 \%)$ & 0.540 & 0.664 \\
\hline Prediabetes & $101(25.7 \%)$ & $61(26.6 \%)$ & $21(22.1 \%)$ & $19(27.5 \%)$ & & \\
\hline Diabetes & $54(13.7 \%)$ & $28(12.2 \%)$ & $13(13.7 \%)$ & $13(18.8 \%)$ & & \\
\hline Total cholesterol (mg/dl) & $217.7 \pm 36.5$ & $213.8 \pm 35.5$ & $221.2 \pm 40.0$ & $225.9 \pm 33.5$ & 0.030 & 0.031 \\
\hline HDL cholesterol (mg/dl) & $61.9 \pm 17.6$ & $62.5 \pm 17.8$ & $63.4 \pm 18.6$ & $57.5 \pm 15.0$ & 0.073 & 0.076 \\
\hline LDL cholesterol (mg(dl) & $139.4 \pm 33.0$ & $135.2 \pm 32.9$ & $142.2 \pm 34.0$ & $149.8 \pm 29.3$ & 0.003 & 0.002 \\
\hline Triglycerides (mg/dl) & $132.0 \pm 85.3$ & $129.2 \pm 92.1$ & $125.7 \pm 78.4$ & $149.8 \pm 68.2$ & 0.153 & 0.159 \\
\hline Systolic BP (mmHg) & $120.7 \pm 16.8$ & $118.4 \pm 16.1$ & $120.9 \pm 18.7$ & $128.2 \pm 14.1$ & $<0.001$ & $<0.001$ \\
\hline Diastolic BP (mmHg) & $75.3 \pm 10.1$ & $75.0 \pm 9.2$ & $74.5 \pm 12.0$ & $77.6 \pm 9.6$ & 0.112 & 0.120 \\
\hline Antihypertensive medication & $101(25.7 \%)$ & $43(18.8 \%)$ & $27(28.4 \%)$ & $31(44.9 \%)$ & $<0.001$ & $<0.001$ \\
\hline Physically active & $234(59.5 \%)$ & $135(59.0 \%)$ & $55(57.9 \%)$ & $44(63.8 \%)$ & 0.722 & 1 \\
\hline \multicolumn{7}{|l|}{ Alcohol consumption } \\
\hline No & $94(23.9 \%)$ & $57(24.9 \%)$ & $20(21.1 \%)$ & $17(24.6 \%)$ & 0.308 & 0.313 \\
\hline Moderate & $222(56.5 \%)$ & $135(59.0 \%)$ & $53(55.8 \%)$ & $34(49.3 \%)$ & & \\
\hline Heavy & $77(19.6 \%)$ & $37(16.2 \%)$ & $22(23.2 \%)$ & $18(26.1 \%)$ & & \\
\hline NSAID & $14(3.6 \%)$ & $6(2.6 \%)$ & $5(5.3 \%)$ & $3(4.3 \%)$ & 0.410 & 0.874 \\
\hline Antithrombotic medication & $23(5.9 \%)$ & $6(2.6 \%)$ & $11(11.6 \%)$ & $6(8.7 \%)$ & 0.004 & 0.115 \\
\hline
\end{tabular}

Data are means and standard deviations for continuous variables and counts and percentages for categorical variables

*Comparison between subjects with advanced and no diverticular disease

Referring to laboratory results, total cholesterol and lowdensity-lipoprotein (LDL) cholesterol were significantly elevated in subjects with diverticular disease $(p<0.03)$. Additionally, we found elevated systolic blood pressure and a higher proportion of intake of antihypertensive medication in subjects with diverticular disease with a presence of diverticula in the colon (all $p<0.001$ ). We did not find significant differences regarding diabetes status or lifestyle factors such as smoking and alcohol consumption as well as physical activity between subjects with and without diverticular disease (all $p>0.3$ ). Further demographics and risk profiles are provided in Table 1.

Comparing participants with diverticular disease only detected in the right-sided $(n=12)$ versus left-sided colonic segments $(n=129)$, subjects with left-sided diverticular disease were older and had significantly higher BMI levels (mean age: $51.4 \pm 9.5$ vs. $58.9 \pm 8.9$ years, mean BMI: $25.6 \pm 4.9$ vs. $28.8 \pm 5.0 \mathrm{~kg} / \mathrm{m}^{2}$, for subjects with right-sided vs. right-sided diverticular disease, respectively; all $p \leq 0.04)$. Furthermore, subjects with left-sided diverticular disease presented higher levels of triglycerides and systolic blood pressure than those with right-sided diverticular disease (triglycerides: $138.1 \pm$ 76.5 vs. $92.2 \pm 26.1 \mathrm{mg} / \mathrm{dl}$ and systolic blood pressure: $124.5 \pm 18.1$ vs. $110.6 \pm 11.8 \mathrm{mmHg}$ for left-sided vs. rightsided diverticular disease, respectively; all $p \leq 0.04$ ).

Comparing no or mild grade diverticular disease with advanced grade diverticular disease, age (OR: $2.24 ; 95 \% \mathrm{CI}$ : $[1.60,3.15], p<0.001$ ), BMI (OR: 1.44; 95\% CI: [1.06, 1.96], $p=0.02)$ and LDL cholesterol (OR: $2.35,95 \% \mathrm{CI}$ : $[1.09,5.06], p=0.03)$ were associated with the presence of advanced grade diverticular disease. 


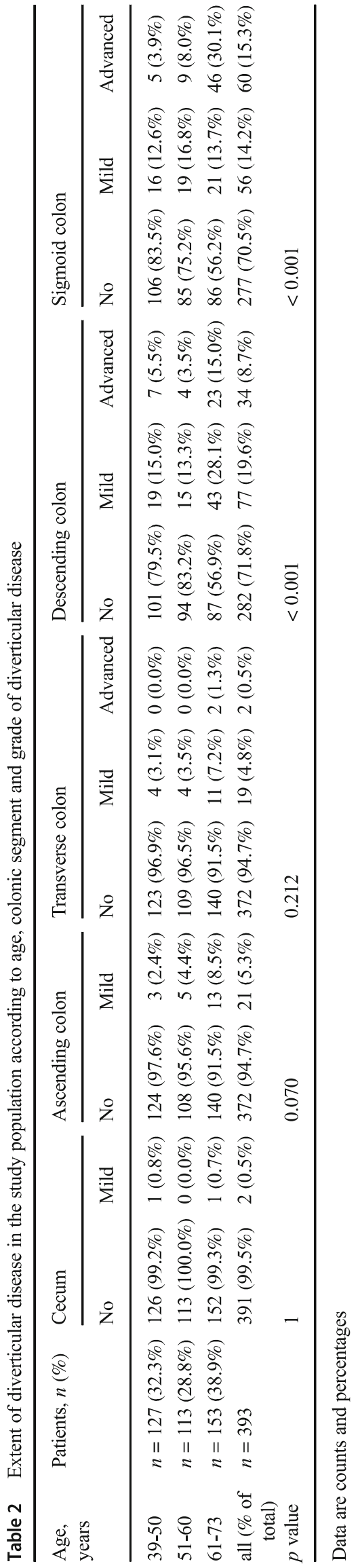

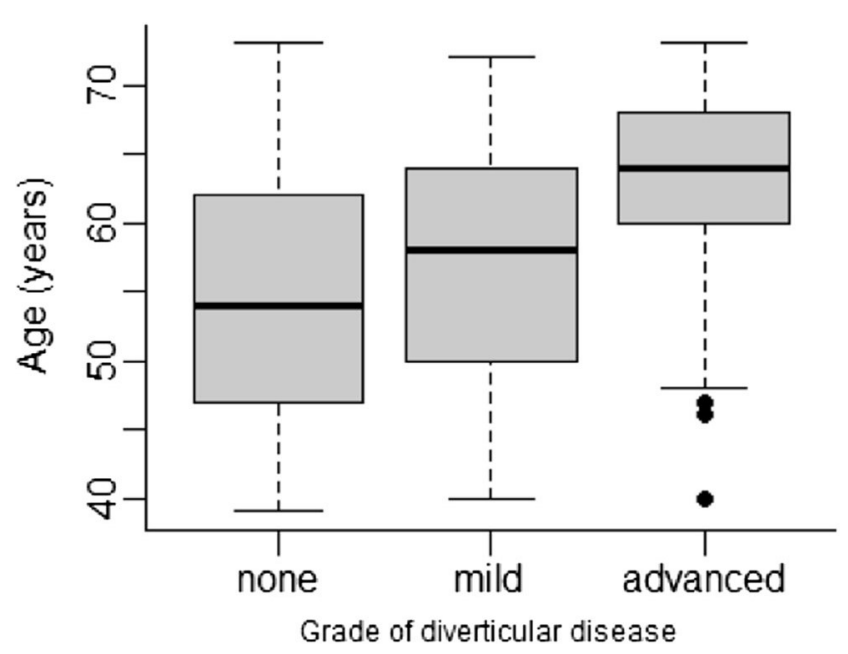

Fig. 4 Grade of diverticular disease according to age

\section{Discussion}

In this study, we provide initial data on the presence and extent of diverticular disease in an asymptomatic, population-based study cohort using MRI as the primary imaging modality. Our findings demonstrate a prevalence of $42 \%$ for asymptomatic diverticular disease in a Western population, affecting predominantly the descending and sigmoid colon and being associated with higher age and cardiometabolic risk factors such as BMI and LDL cholesterol. Furthermore, our results indicate that MRI may be an appropriate non-invasive modality for the assessment and characterization of diverticular disease with an excellent intra- and inter-rater reliability, revealing detailed insight into the pathomechanism of asymptomatic diverticular disease in a general Western population. The results of our study are generalizable to an asymptomatic general population without prior cardiovascular disease in a preventive setting in whom MRI can be performed without contraindications [19].

Although diverticular disease confers several risk factors such as bleeding and inflammation, the exact prevalence of

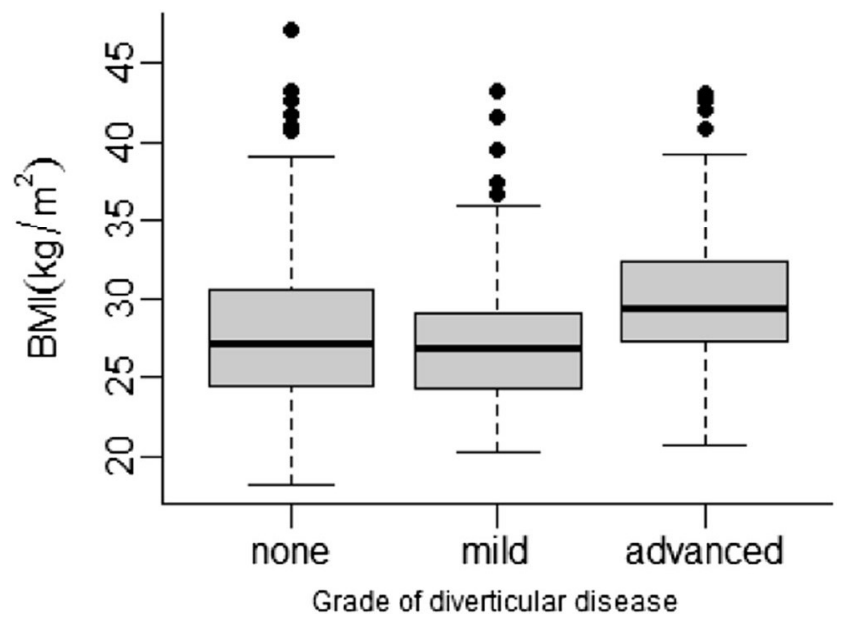

Fig. 5 Grade of diverticular disease according to BMI 
presupposed asymptomatic diverticular disease in a general population is unknown [4]. Colonoscopy represents the current gold standard for the detection of diverticular disease. Previous studies showed that CT and CTC represent noninvasive modalities with excellent diagnostic accuracy, especially in symptomatic diverticular disease [26]. Similarly to CT, MRI was previously shown to provide high sensitivity and specificity for the diagnosis of colonic inflammatory bowel disease including symptomatic diverticular disease [14]. However, data about asymptomatic diverticular disease are very limited, and research regarding the detection of diverticula in the colon assessed by MRI is missing. Thus, detailed insight into the pathophysiologic mechanism and association of asymptomatic and symptomatic diverticular disease is lacking.

Most data on the prevalence of asymptomatic diverticular disease are based on incidental findings from invasive colonoscopy or CTC in a limited number of patients as well as from postmortem surveys. For example, Everhart et al found a prevalence rate ranging from $32 \%$ to $71 \%$ according to age in a colonoscopy study [5]. De Cecco et al recently evaluated the extent and distribution of asymptomatic diverticular disease in a large population including 1091 participants, assessed by CTC, and found an overall prevalence of $47 \%$ [25]. These findings are in line with our study results, as we found an overall prevalence of $42 \%$ for diverticular disease in our study population. Although this was a clinical cohort of mostly asymptomatic subjects, we confirm the relatively high prevalence and extend these findings to the general population. Also, we confirm the predominance of diverticula to the descending and sigmoid colon [7, 25, 27]. Interestingly, this is in contrast to earlier research stemming from Asian populations, in which a higher prevalence of diverticular disease of the right-sided colon has been observed. The fact that our population, representing Caucasian individuals only, exhibits a more right-sided diverticulosis supports the hypothesis of racial variation, assuming that left-sided diverticular disease is more common in the Western population $[6,7]$. It can be anticipated that these racial differences will also translate into the identification of genetic loci associated with diverticular disease if imaging is implemented into larger-scale population-based studies, such as the German National Cohort or UK Biobank [28, 29].

Interestingly, our results showed no significant gender difference, which adds to the ongoing controversy on the gender dependency of diverticular disease. Yamamichi et al and Peery et al found a preponderance of asymptomatic diverticular disease in males in a Japanese and American population, respectively $[6,27]$. Along with our results, De Cecco et al found no significant difference between male and female participants in subjects with asymptomatic diverticular disease [25]. Contrarily, several former studies reported a higher prevalence of hospitalization due to diverticular disease in women [30,
31]. More outcome-related research is needed to determine whether gender serves as an effect modifier for the clinical manifestation of acute disease.

In addition, we found that age is a strong predictor of the presence and extent of diverticular disease. This is in line with earlier studies demonstrating that diverticular disease is more common in the elderly, also in an asymptomatic stage [5-7, 25]. Furthermore, comparing participants with only right-sided diverticular disease with those with only left-sided diverticular disease, the latter group had significantly older subjects with higher levels of BMI, triglycerides and systolic blood pressure in our study. Additionally, as we found an association of the extent and distribution of diverticular disease in the left-sided colonic segments according to increasing age, our results support the assumption that most of the diverticula recorded in the descending or sigmoid colon seem to be rather acquired than congenital. Furthermore, left-sided diverticular disease seems to be strongly associated with age and metabolic risk factors, and there seems to be hereditary as well as pathophysiological differences concerning the development and distribution of diverticular disease in the colonic segments $[27,32$, 33]. However, as mentioned above, previous research showed that right-sided diverticular disease in Western populations does not seem to be such an uncommon finding as initially presumed [25]. Thus, further large-scale population-based research is warranted.

Our study results showed that increased BMI and increased systolic blood pressure and high LDL cholesterol levels are associated with diverticular disease. In a retrospective casecontrol study including participants who underwent colonoscopy, Kopylov et al found that asymptomatic diverticular disease was associated with obesity [34]. In a large Swedish 28year follow-up study, obesity was found to be strongly associated with severe diverticular disease leading to hospitalization, and a prospective US cohort study was in line with these findings, reporting an association of obesity and consequential severe diverticular disease $[3,35]$. However, although higher BMI was found to be significantly associated with mild or adverse diverticular disease in our study, intervals between the several grades of diverticular disease were rather lower than expected (Fig. 5), indicating a differentiating pathophysiologic mechanism of the development of asymptomatic compared with symptomatic diverticular disease. Furthermore, while we observed some associations after multivariate adjustment that are not clearly related to diverticular disease (i.e., LDL or cholesterol) and may suggest further residual confounding, it is likely that these are factors in a complex cardiometabolic risk type that may warrant more detailed analysis in subsequent studies.

Several studies reported an additional association of diverticular disease with alcohol consumption, smoking and diabetes. However, we found no significant association between the 
presence of diverticular disease and these cardiometabolic risk factors $[6,7,9,36]$. One explanation may be that our study population appears to be rather health-conscious, and participants with heavy smoking habits or alcohol consumption were scarce. Also, we report initial observations from a sample from the general population, which per se differs significantly from clinical cohorts and may exhibit different characteristics that may be more relevant in a preventive setting. However, further confirmatory research is mandatory. This may be particularly relevant as in symptomatic diverticular disease a higher risk of complications is predicted by smoking, increased alcohol consumption, obesity and physical inactivity $[9,37]$. For instance, Crowe et al found an increased risk of hospitalization in smoking patients compared with nonsmokers [8]. In prior work, alcohol consumption was a predictive factor for severe and complicated diverticular disease, but this association was confounded by smoking status, which may highlight the important role of smoking as a major complicating risk factor in diverticular disease [8, 37, 38].

Previous studies found that physical inactivity promotes the risk of inflammatory diverticular disease and, conversely, that physical activity prevented diverticular disease and hospitalization [39-42]. Our results showed a higher prevalence of diverticular disease in obese subjects; however, there was no significant correlation between the presence of colonic diverticula and physical activity. One potential explanation may be that our assessment of physical activity was self-reported with well-known limitations, thus, more detailed research is justified.

Our study has several limitations. Information about the outcome is missing; thus, we are not able to provide risk estimates on the prognostic relevance of our MRI findings as the present analysis represents a baseline cross-sectional analysis of the study sample. Due to the prospective design of the study, all imaging parameters were prospectively defined; however, given the exploratory nature of the analysis, no dedicated sample size calculation was performed. Furthermore, the study was designed for the assessment of cardiometabolic multiorgan alterations; thus, participants were not prepared for specified gastrointestinal examinations and did not receive preparations such as a fiber-free diet, bowel cleansing or oral/rectal preparations for fecal tagging prior to the MRI examination. However, while this may decrease the accuracy for detailed morphologic assessment of single colonic diverticula, we first report its prevalence in a sample from the general, asymptomatic population, which precluded more complex and/or invasive preparations. Furthermore, we applied the DIXON VIBE sequence to assess diverticular disease although there are certainly more dedicated sequences with higher resolution imaging features available. While these sequences may be more feasible to assess morphologic details, the applied DIXON VIBE sequence allows for a feasible combination of morphologic resolution and acquisition time in the setting of whole-body MRI in population-based study populations. As such, we were not able to prove the accuracy of MRI by including an established gold standard such as colonoscopy. However, we graded diverticular disease in broader categories, which may have attenuated inaccuracies inherent to MRI. Also, we found excellent inter- and intra-rater reliability, but further studies comparing the detection of diverticula assessed by MRI with findings in colonoscopy are warranted.

In conclusion, MRI is a reproducible, low-risk imaging modality for the assessment of the presence and extent of diverticular disease in the general population. Further research to elucidate the role of individual predisposition and risk of symptomatic diverticular disease and its complications in larger cohorts is warranted.

Funding This study was funded by the German Research Foundation (DFG, Bonn, Germany), the German Centre for Cardiovascular Disease Research (DZHK, Berlin, Germany) and the German Centre for Diabetes Research (DZD e.V., Neuherberg, Germany).

The KORA study was initiated and financed by the Helmholtz Zentrum München-German Research Center for Environmental Health, which is funded by the German Federal Ministry of Education and Research (BMBF) and by the State of Bavaria.

\section{Compliance with ethical standards}

Guarantor The scientific guarantor of this publication is Fabian Bamberg, MD, MPH.

Conflict of interest The authors of this manuscript declare no relationships with any companies, whose products or services may be related to the subject matter of the article.

Statistics and biometry Two of the authors (SR, RL) have significant statistical expertise.

Informed consent Written informed consent was obtained from all subjects in this study.

Ethical approval Institutional Review Board approval was obtained.

Methodology

- prospective

- population based

- case-control study

- performed at one institution

\section{References}

1. Böhm SK (2015) Risk factors for diverticulosis, diverticulitis, diverticular perforation, and bleeding: a plea for more subtle history taking. Viszeralmedizin 31:84-94

2. Feuerstein JD, Falchuk KR (2016) Diverticulosis and diverticulitis. Mayo Clin Proc 91:1094-1104

3. Strate LL, Liu YL, Aldoori WH, Syngal S, Giovannucci EL (2009) Obesity increases the risks of diverticulitis and diverticular bleeding. Gastroenterology 136(115-122):e111 
4. Stollman N, Raskin JB (2004) Diverticular disease of the colon. Lancet 363:631-639

5. Everhart JE, Ruhl CE (2009) Burden of digestive diseases in the United States part II: lower gastrointestinal diseases. Gastroenterology 136:741-754

6. Yamamichi N, Shimamoto T, Takahashi Y et al (2015) Trend and risk factors of diverticulosis in Japan: age, gender, and lifestyle/ metabolic-related factors may cooperatively affect on the colorectal diverticula formation. PLoS One 10:e0123688

7. Wang FW, Chuang HY, Tu MS et al (2015) Prevalence and risk factors of asymptomatic colorectal diverticulosis in Taiwan. BMC Gastroenterol 15:40

8. Crowe FL, Appleby PN, Allen NE, Key TJ (2011) Diet and risk of diverticular disease in Oxford cohort of European Prospective Investigation into Cancer and Nutrition (EPIC): prospective study of British vegetarians and non-vegetarians. BMJ 343:d4131

9. Usai P, Ibba I, Lai $\mathrm{M}$ et al (2011) Cigarette smoking and appendectomy: effect on clinical course of diverticulosis. Dig Liver Dis 43:98-101

10. Connelly TM, Berg AS, Hegarty JP et al (2014) The TNFSF15 gene single nucleotide polymorphism rs 7848647 is associated with surgical diverticulitis. Ann Surg 259:1132-1137

11. Granlund J, Svensson T, Olén O et al (2012) The genetic influence on diverticular disease-a twin study. Aliment Pharmacol Ther 35: 1103-1107

12. Weizman AV, Nguyen GC (2011) Diverticular disease: epidemiology and management. Can J Gastroenterol 25:385-389

13. Shahedi K, Fuller G, Bolus R et al (2013) Long-term risk of acute diverticulitis among patients with incidental diverticulosis found during colonoscopy. Clin Gastroenterol Hepatol 11:1609-1613

14. Heverhagen JT, Sitter H, Zielke A, Klose KJ (2008) Prospective evaluation of the value of magnetic resonance imaging in suspected acute sigmoid diverticulitis. Dis Colon Rectum 51:1810-1815

15. Buckley O, Geoghegan T, McAuley G, Persaud T, Khosa F, Torreggiani WC (2007) Pictorial review: magnetic resonance imaging of colonic diverticulitis. Eur Radiol 17:221-227

16. Hammond NA, Miller FH, Yaghmai V, Grundhoefer D, Nikolaidis P (2008) MR imaging of acute bowel pathology: a pictorial review. Emerg Radiol 15:99-104

17. Heverhagen JT, Ishaque N, Zielke A et al (2001) Feasibility of MRI in the diagnosis of acute diverticulitis: initial results. MAGMA 12:4-9

18. Heverhagen JT, Zielke A, Ishaque N, Bohrer T, El-Sheik M, Klose KJ (2001) Acute colonic diverticulitis: visualization in magnetic resonance imaging. Magn Reson Imaging 19:1275-1277

19. Bamberg F, Hetterich H, Rospleszcz S et al (2017) Subclinical disease burden as assessed by whole-body MRI in subjects with prediabetes, subjects with diabetes, and normal control subjects from the general population: The KORA-MRI Study. Diabetes 66:158-169

20. Holle R, Happich M, Löwel H, Wichmann HE, Group MKS (2005) KORA - a research platform for population based health research. Gesundheitswesen 67(Suppl 1):S19-S25

21. Ruf E, Baumert J, Meisinger C, Döring A, Ladwig KH; MONICA/ KORA investigators (2014) Are psychosocial stressors associated with the relationship of alcohol consumption and all-cause mortality? BMC Public Health 14:312

22. Authors/Task Force Members, Rydén L, Grant PJ et al (2013) ESC Guidelines on diabetes, pre-diabetes, and cardiovascular diseases developed in collaboration with the EASD: the Task Force on diabetes, pre-diabetes, and cardiovascular diseases of the European Society of Cardiology (ESC) and developed in collaboration with the European Association for the Study of Diabetes (EASD). Eur Heart J 34:3035-3087

23. World Health Organization (2006) Definition and diagnosis of diabetes mellitus and intermediate hyperglycemia. In: Press W (ed) Publications of the World Health Organization. World Health Organization, Geneva

24. Gray H (2013) Gray's anatomy. Fall River Press, New York

25. De Cecco CN, Ciolina M, Annibale B et al (2016) Prevalence and distribution of colonic diverticula assessed with CT colonography (CTC). Eur Radiol 26:639-645

26. Peery AF (2016) Recent advances in diverticular disease. Curr Gastroenterol Rep 18:37

27. Peery AF, Keku TO, Martin CF et al (2016) Distribution and characteristics of colonic diverticula in a United States screening population. Clin Gastroenterol Hepatol 14(980-985):e981

28. Bamberg F, Kauczor HU, Weckbach S et al (2015) Whole-body MR imaging in the German National Cohort: rationale, design, and technical background. Radiology 277:206-220

29. Fry A, Littlejohns TJ, Sudlow C et al (2017) Comparison of sociodemographic and health-related characteristics of UK Biobank participants with those of the general population. Am J Epidemiol 186:1026-1034

30. Wheat CL, Strate LL (2016) Trends in hospitalization for diverticulitis and diverticular bleeding in the United States From 2000 to 2010. Clin Gastroenterol Hepatol 14(96-103):e101

31. Warner E, Crighton EJ, Moineddin R, Mamdani M, Upshur R (2007) Fourteen-year study of hospital admissions for diverticular disease in Ontario. Can J Gastroenterol 21:97-99

32. Hughes LE (1969) Postmortem survey of diverticular disease of the colon. I. Diverticulosis and diverticulitis. Gut 10:336-344

33. Radhi JM, Ramsay JA, Boutross-Tadross O (2011) Diverticular disease of the right colon. BMC Res Notes 4:383

34. Kopylov U, Ben-Horin S, Lahat A, Segev S, Avidan B, Carter D (2012) Obesity, metabolic syndrome and the risk of development of colonic diverticulosis. Digestion 86:201-205

35. Rosemar A, Angerås U, Rosengren A (2008) Body mass index and diverticular disease: a 28 -year follow-up study in men. Dis Colon Rectum 51:450-455

36. Sharara AI, El-Halabi MM, Mansour NM et al (2013) Alcohol consumption is a risk factor for colonic diverticulosis. J Clin Gastroenterol 47:420-425

37. Papagrigoriadis S, Macey L, Bourantas N, Rennie JA (1999) Smoking may be associated with complications in diverticular disease. Br J Surg 86:923-926

38. Tønnesen H, Engholm G, Moller H (1999) Association between alcoholism and diverticulitis. Br J Surg 86:1067-1068

39. Williams PT (2009) Incident diverticular disease is inversely related to vigorous physical activity. Med Sci Sports Exerc 41:1042-1047

40. Aldoori WH, Giovannucci EL, Rimm EB et al (1995) Prospective study of physical activity and the risk of symptomatic diverticular disease in men. Gut 36:276-282

41. Strate LL, Liu YL, Aldoori WH, Giovannucci EL (2009) Physical activity decreases diverticular complications. Am J Gastroenterol 104:1221-1230

42. Hjern F, Wolk A, Håkansson N (2012) Obesity, physical inactivity, and colonic diverticular disease requiring hospitalization in women: a prospective cohort study. Am J Gastroenterol 107:296-302 